
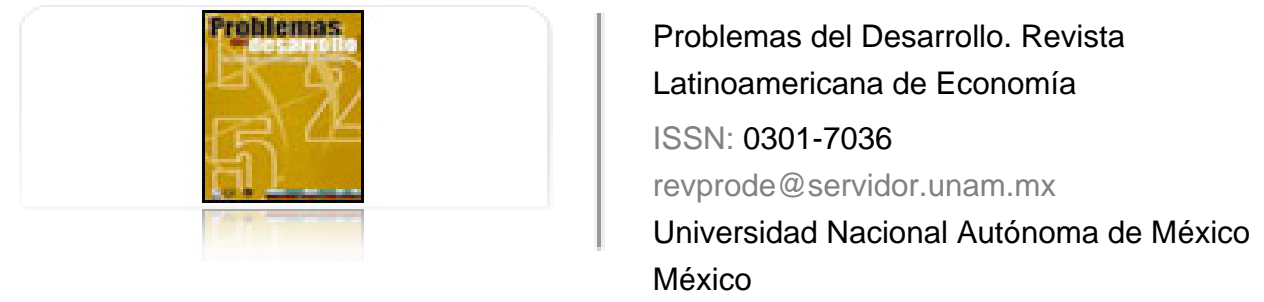

Caballero Güendolain, Karina; Galindo Paliza, Luis Miguel El consumo de energía en México y sus efectos en el producto y los precios Problemas del Desarrollo. Revista Latinoamericana de Economía, vol. 38, núm. 148, enero-marzo, 2007, pp. 127-151

Universidad Nacional Autónoma de México

Distrito Federal, México

Disponible en: http://www.redalyc.org/articulo.oa?id=11820155006

Cómo citar el artículo

- Número completo

- Más información del artículo

- Página de la revista en redalyc.org

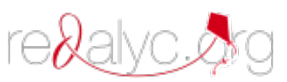

Sistema de Información Científica

Red de Revistas Científicas de América Latina, el Caribe, España y Portugal Proyecto académico sin fines de lucro, desarrollado bajo la iniciativa de acceso abierto 


\section{El CONSUmo DE ENERGÍA en MÉXICO Y SUS EFECTOS}

EN EL PRODUCTO Y LOS PRECIOS

\section{Karina Caballero Güendolain* Luis Miguel Galindo Paliza**}

Fecha de recepción: 8 de junio de 2006. Fecha de aceptación: 18 de octubre de 2006.

\section{Resumen}

El principal objetivo de este trabajo es analizar las relaciones entre el consumo de energía y el producto y los precios en la economía mexicana. Los resultados más importantes indican que dicho consumo está estrechamente asociado a la evolución del producto y mantiene una relación negativa pero inelástica con el precio de la energía. Asimismo, ante un aumento de los precios de ésta, se encontró que el producto disminuye y el nivel de precios generales aumenta. Esta interdependencia entre variables macroeconómicas y precios de la energía indica que un aumento de estos últimos es insuficiente para controlar el consumo de energía, lo cual limita el uso de los precios como instrumento único de política pública. Este escenario dificulta la opción de desacoplar la trayectoria del consumo de energía y el producto en los próximos años.

Palabras clave: energía, producto, precios, cointegración, estabilidad estructural.

* Técnico académico, titular de tiempo completo, Facultad de Economía, unAM. Correo electrónico: karinacg@economia.unam.mx y karina.caballero@gmail.com

** Profesor titular C de tiempo completo en la Facultad de Economía, UNAM. Correo electrónico: gapaliza@servidor.unam.mx 
Abstract

The paper's main object is to analyze the relationships between energy consumption, production and prices in the Mexican economy. The relevant results indicate that consumption is closely related to the movement of production and has a negative but inelastic relationship with the price of energy. At the same time, given the rise in energy prices, it was found that production falls as the general price level increases. This interdependence between macroeconomic variables and energy prices indicates that an increase in the latter is insufficient to control energy consumption, thus limiting the use of prices as a unique public policy instrument. This scenario makes it hard to consider the option of decoupling the trajectory of energy consumption and production in coming years.

Key words: energy, production, prices, co-integration, structural stability.

\section{Résumé}

Le principal objectif de ce travail est d'analyser les relations qui unissent la consommation d'énergie au produit et aux prix dans l'économie mexicaine. Les résultats les plus importants indiquent que la dite consommation est étroitement liée à l'évolution du produit et maintient une relation négative mais inflexible avec le prix de l'énergie. Ainsi, devant une augmentation des prix de celle-ci, il est apparu que le produit diminue et le niveau des prix généraux augmente. Cette interdépendance entre variables macroéconomiques et les prix de l'énergie indique qu'une augmentation de ces derniers est insuffisante pour contrôler sa consommation, ce qui limite la portée du seul recours aux prix comme instrument de politique publique. Ce contexte rend difficile la perspective d'un désaccouplement de la trajectoire de la consommation d'énergie et de celle du produit dans les prochaines années.

Mots clés: énergie, produit, prix, co-intégration, stabilité structurelle.

\section{Resumo}

O principal objetivo deste trabalho é analisar as relações entre o consumo de energia e o produto e os preços na economia mexicana. Os resultados mais importantes indicam que tal consumo está estreitamente associado à evolução do produto e mantém uma relação negativa mas inelástica com o preço da energia. Da mesma forma, perante um aumento dos preços da mesma, achou-se que o produto diminui e o nível de preços gerais aumenta. Esta interdependência entre variáveis macroeconômicas e preços da energia indica que um aumento destes últimos é insuficiente para controlar o consumo de energia, o qual limita o uso dos preços como instrumento único de política pública. Este cenário dificulta considerar a opção de desacoplar a trajetória do consumo de energia e o produto nos próximos anos.

Palavras chave: energia, produto, preços, cointegração, estabilidade estrutural. 


\section{Introducción}

1

os actuales niveles de consumo de energía en México asociados, fundamentalmente, a la trayectoria del producto son inconsistentes con un desarrollo económico sustentable. En efecto, los requerimientos energéticos de la economía mexicana por unidad de producto son ciertamente elevados, ${ }^{1}$ de tal modo que una recuperación sostenida del ritmo de crecimiento económico se vería acompañada por una creciente demanda de energía con consecuencias negativas para el ambiente. En particular, desde el punto de vista ambiental destacan dos efectos dañinos en los niveles actuales de consumo energético: en primer lugar, la oferta actual de energía en México se basa, en su mayoría, en el uso de recursos no renovables, como el petróleo. En ese sentido, las condiciones de su consumo actual son inconsistentes con las definiciones de sustentabilidad débil y fuerte o la regla de Hartwick (Hartwick, 1977; Pearce et al., 1990; Gutés, 1996; Pearce y Atkinson, 1995). En segundo lugar, las emisiones a la atmósfera asociadas al consumo de energía tienen consecuencias negativas, tales como contaminación atmosférica y emisión de gases de efecto invernadero, asociados al cambio climático que inciden negativamente sobre la salud de la población y la sustentabilidad de diversos ecosistemas.

En este contexto, es fundamental considerar las posibilidades reales para desacoplar la trayectoria del consumo de energía a la del producto y evaluar la pertinencia de la discusión reciente sobre el "alto precio de la energía en México". En particular, es posible analizar e identificar escenarios alternativos de consumo energético, así como sus relaciones con el producto y los precios, y con posibles opciones de innovación tecnológica y su impacto agregado en el producto y los precios. Así, el principal objetivo de este trabajo es analizar, desde el punto de vista económico, los efectos de distintos escenarios de crecimiento económico y de políticas de precios para la energía y sus efectos agregados en el producto y los precios en la economía mexicana. En particular, se identifican varios escenarios considerando las posibilidades para desacoplar la trayectoria del consumo de energía y del producto.

1 De acuerdo con el Programa de Desarrollo de Naciones Unidas, México es uno de los países más intensivos en el uso de la energía, por ejemplo, por cada dólar se emplean 8.7 megajoules; en cambio, en Estados Unidos, Japón, China y la Unión Europea por cada dólar se utilizan 10 megajoules en promedio (Aguayo y Gallagher, 2005). 
El trabajo se divide en cuatro secciones. En la primera se exploran los diferentes marcos teóricos en los cuales se han modelado las cuestiones energéticas; enseguida se detalla la metodología econométrica. La tercera sección contiene la evidencia empírica, resultado de un enfoque de arriba hacia abajo (top-down) con un modelo econométrico de tres ecuaciones base, una de consumo de energía, una de producto y finalmente una de precios. Con base en éstas se realizaron pronósticos y diversos escenarios de simulación, con objeto de identificar el impacto del aumento de los precios relativos de la energía en las demás variables. La última sección presenta las conclusiones y examina las posibles políticas energéticas que induzcan un desarrollo sustentable, tomando como referencia los resultados obtenidos del modelo.

\section{Marco general y metodología econométrica}

El análisis de la relación entre el consumo de energía, el producto y los precios es uno de los temas fundamentales para determinar las condiciones de un desarrollo económico sustentable. En el análisis económico, las relaciones entre el consumo de energía, el producto y los precios han sido estudiadas desde diversos puntos de vista. En ese sentido, al menos destacan dos enfoques que implican diversas opciones de análisis. Así, por un lado, tenemos uno conocido como bottom up, orientado más al uso de modelos basados en estudios de ingeniería donde se utilizan directamente los requerimientos energéticos del equipo disponible asociado a tecnologías específicas (Böhringer, 1998; Graham y Williams, 2003). Por otro lado, se tiene un enfoque asociado a los modelos top down, donde el consumo de energía se analiza en el contexto de un modelo macroeconómico, ya sea del tipo de los modelos de equilibrio general computable o de un modelo econométrico parcial (Mabey, Hall, Smith y Gupta, 1997; Jorgenson y Wilcoxen, 1993a; Jorgenson, 1984; Jorgenson y Wilcoxen, 1998; Ho y Jorgenson, 1998, y Pyndick, 1979). Recientemente, se observa un creciente interés por combinar esos tipos de modelos para obtener estimaciones más precisas y simulaciones históricas más representativas (véase, por ejemplo, Mabey, Hall, Smith y Gupta, 1997:70, o Jorgenson, 1998). Esto es, los modelos de equilibrio general utilizan un modelo teórico bien definido fundado en un comportamiento optimizador de los agentes individuales, los cuales normalmente son calibrados para un año base mediante algunos parámetros clave asociados a las elasticidades ingreso o precio de la energía. En ese sentido, esos modelos disponen de gran elegancia y consistencia teórica, pero por desgracia suponen como conocido un conjunto de información empírica que es escasa, particularmente en los países en 
vías de desarrollo. En el otro extremo, los modelos estimados econométricamente pierden consistencia teórica, pero tienen mayor fortaleza debido al uso de la evidencia empírica disponible.

En este trabajo se optó, entonces, por estimar econométricamente esos modelos para identificar con precisión las relaciones que se establecen entre las variables. Así, tenemos el modelo estimado que consiste en tres ecuaciones base. La primera representa una demanda de energía tradicional (Galindo y Sánchez, 2005; Mabey, Hall, Smith y Gupta, 1997; Jorgenson, 1998), donde el consumo de energía depende del ingreso y de su precio relativo (Pindyck, 1979). En este contexto, es común la simplificación de suponer el consumo de energía como una entidad derivada de la trayectoria del producto y de su composición estructural, del progreso técnico y de la evolución de los precios relativos (Mabey, Hall, Smith y Gupta, 1997; Howarth, Schipper y Anderson, 1993; Nachane, Nadkarni y Karnik, 1988, y Jorgenson, 1998).

La ecuación 2 representa una especificación para el producto. En este caso, se considera que el crecimiento económico se asocia, fundamentalmente, ${ }^{2}$ a la evolución de la inversión (King y Levine, 1994; Obsfeld y Rogoff, 1999), apoyada por los efectos del tipo de cambio real (Garcés, 2003), los efectos de la dinámica económica del resto del mundo (Guerrero, 2004), usando el producto de Estados Unidos como una aproximación (Garcés, 2003), y a la posible presencia de procesos de convergencia o de transmisión del crecimiento económico (Sala-i-Martin 1996). Asimismo, los efectos de los precios relativos de la energía se consideran originados dado que ésta es un insumo fundamental para la producción, de modo que un aumento de su precio tiene efectos negativos en el producto (Jorgenson y Wilcoxen, 1998).

Finamente, la tercera ecuación determina al nivel de precios general. La especificación utilizada incluye las presiones de costos internos y externos y una validación de la tasa de inflación. Así, los principales determinantes del nivel de precios en México son: el tipo de cambio nominal o pass trought (Schmidt-Hebbel y Werner, 2002; Gil Díaz y Ramos Tercero, 1988), el salario mínimo nominal (Garcés, 2002), los precios relativos de la energía y el agregado monetario M2 como mecanismo de validación del ritmo de crecimiento de los precios.

De esta manera, las ecuaciones base se especificaron de la siguiente forma:

2 Existen desde luego otros factores tales como distribución del ingreso, capital humano o estado de derecho (véase Ros, 2000). 


$$
\begin{aligned}
& D_{t}=F\left(Y_{t}, P R E_{t}\right) \\
& Y_{t}=F\left(I_{t}, T_{t}, X_{t}, P R E_{t}\right) \\
& P_{t}=\left(S_{t}, W_{t}, M_{t}, P R E_{t}\right)
\end{aligned}
$$

Las estimaciones econométricas se realizaron atendiendo el orden de integración de las series, la cointegración y el concepto de mecanismo de corrección de errores (véase, por ejemplo, Maddala y Kim, 1998). De ese modo, se procedió a identificar el orden integración de las series con base en las pruebas de DickeyFuller Aumentada (ADF) (1981), de Phillips-Perron (PP) (1988) y de Kwiatkowsky et al. (KPSS) (1992). En las pruebas ADF y PP se utiliza el procedimiento de lo "general a lo específico", incluyendo inicialmente constante y tendencia, además de evaluar su significancia estadística. El número de rezagos se seleccionó utilizando el procedimiento conocido como t-sig (Ng y Perron, 1995), tomando al comienzo un número de rezagos igual a 4 , reduciéndolo hasta obtener un término estadísticamente significativo.

El análisis de cointegración se basó en el procedimiento de Johansen (1988), donde el modelo de vectores autoregresivos (VAR) se representa como (Johansen, 1995):

$$
X_{t}=A_{1} X_{t-1}+\ldots+A_{k} X_{t-k}+\Phi D_{t}+u_{t}
$$

en el cual $X_{t}$ representa a un vector que incluye a todas las variables, $A_{1}, \ldots, A_{k}$ son matrices que incluyen a los coeficientes estimados; $D_{t}$ puede incluir una constante, tendencia y dummies estacionales o de intervención, $\mathrm{y}_{\mathrm{t}}$ es el término de error con media cero y varianza constante. En este caso, bajo la presencia de cointegración, el VAR puede reescribirse en su forma de corrección de error conocida como VECM (Johansen, 1988 y 1995):

$$
\Delta \mathrm{X}_{\mathrm{t}}=\Gamma_{1} \Delta \mathrm{X}_{\mathrm{t}-1}+\Gamma_{2} \Delta \mathrm{X}_{\mathrm{t}-2}+\ldots+\Gamma_{\mathrm{k}-1} \Delta \mathrm{X}_{\mathrm{t}-\mathrm{k}+1}-\Pi \mathrm{X}_{\mathrm{t}-1}+\Phi \Delta_{\mathrm{t}}+\mathrm{u}_{\mathrm{t}}(5)
$$

en la cual $\Pi=-\mathrm{I}+\sum_{\mathrm{i}=1}^{\mathrm{k}} \mathrm{A}_{\mathrm{i}}$ y $\Gamma \mathrm{j}=-\Sigma_{\mathrm{i}=\mathrm{j}}^{\mathrm{k}} \mathrm{A}_{\mathrm{i}}$ para $\mathrm{j}=1, \ldots \mathrm{k}-1$; bajo la presencia de cointegración se satisface la relación $\Pi=\alpha \beta$ ' (Johansen, 1988). En este caso, los coeficientes del vector $\beta$ sintetizan las relaciones de largo plazo y la matriz $\alpha$ representa la velocidad de ajuste del término de corrección de error. Johansen (1988 y 1995) propuso dos estadísticos para identificar la presencia de la propiedad de cointegración entre las series: el estadístico de la traza (Traza) y el de la raíz carac- 
terística máxima (L-max). Los estadísticos se definen con base en la razón de máxima verosimilitud. Así el estadístico de la traza se representa como:

$$
-2 \ln Q=-T \sum_{i=r+1}^{p} \ln \left(1+\hat{\lambda}_{i}\right)
$$

en el cual $\lambda_{\mathrm{r}+1}, \ldots, \lambda_{\mathrm{p}}$ son estimadas de los $p$ - $r$ menores valores característicos. La hipótesis nula de la prueba asume que el número de vectores de cointegración es menor o igual que $r$, donde $r$ es $0,1,2, \ldots, \mathrm{r}$-p. Alternativamente, el estadístico de la raíz característica máxima se define como:

$$
-2 \ln Q=-T \ln \left(1-\hat{\lambda}_{r-1}\right)
$$

En esta prueba, la hipótesis nula asume $r$ vectores de cointegración con respecto a la alternativa de $r+1$ vectores de cointegración. Los valores críticos del procedimiento de Johansen se ajustaron para poder incluir dummies de intervención de acuerdo con el programa DisCo de Nielsen para evitar los conocidos problemas de la distribución asintótica del estadístico de la traza (Johansen y Nielsen, 1993).

Los vectores de cointegración obtenidos por el procedimiento de Johansen (1988) se utilizaron, entonces, como mecanismo de corrección de errores acorde con el teorema de representación de Engel y Granger (1987) para obtener las ecuaciones econométricas finales. La selección de la especificación del VAR y de las ecuaciones finales se basó en el uso de las pruebas de especificación correcta (Spanos, 1986). ${ }^{3}$

Asimismo, se incluyó el análisis sobre la posible presencia de cambio estructural en las series económicas en el contexto del proceso de cointegración. Esto es, Leybourne y Newbold (2000) y Baffes y Le Valle (2003) argumentan que la presencia de cambio estructural en las series puede traducirse en rechazos espurios en las pruebas de cointegración. De ese modo se procedió a analizar la estabilidad del modelo de cointegración de acuerdo con el procedimiento sugerido por Hansen y Johansen (1993). Así, la estabilidad del rango de cointegración se evaluó acorde con la prueba de la traza. Esto es, el modelo se estimó para una submuestra y posteriormente se añadieron recursivamente más datos para observar la posibilidad de que dicha prueba seleccione un rango distinto o un número diferente de vectores de cointegración. Un valor superior a 1 en la gráfica correspondiente implica el

3 En el caso del var se complementó la selección de acuerdo con el criterio de Hannan y Quinn (1979) que, en promedio, parece ser el más apropiado (Mosconi, 1998). 
rechazo de la hipótesis de estabilidad. Así, el análisis gráfico se basa en dos pruebas: el modelo Z, donde los parámetros de corto y largo plazo son estimados para cada submuestra, y el modelo R, donde los parámetros de corto plazo son considerados fijos y sólo se estiman los que corresponden al ajuste de largo plazo. En el caso de que los resultados entre el modelo $\mathrm{Z}$ y el $\mathrm{R}$ sean contradictorios, se opta por el segundo estadístico (Hansen y Johansen, 1993). Asimismo, debe plantearse que en el caso de la inclusión de dummies de intervención, los residuales pueden ser considerados estacionarios en torno a un componente determinístico (Mosconi, 1998).

Finalmente, se analiza la estabilidad del espacio de cointegración de los coeficientes $\beta\left(\operatorname{Sp}\left(\beta^{*}\right)\right)$ (Mosconi, 1998). En este caso, el modelo es estimado utilizando sólo la primera submuestra de datos bajo la restricción de que el espacio de ésta es igual al de todo el periodo. La hipótesis nula es, entonces, que el espacio es estable y donde la prueba se distribuye como una $X^{2}$ con $p r-r^{2}$ grados de libertad y $p$ es el número filas en $\beta^{*}$ y $r$ es el número de vectores de cointegración.

\section{La evidencia empírica}

La base de datos ${ }^{4}$ utilizada consiste en series anuales para el periodo 1965-2004. El consumo de energía $\left(E_{t}\right)$ corresponde a los datos reportados por la Secretaría de Energía (SENER) del Balance Nacional de Energía medido en pentajoules; el ingreso se aproxima por el producto interno bruto $\left(\mathrm{Y}_{\mathrm{t}}\right)$ a precios de 1993; para la inversión (I) se usó la formación bruta de capital fijo en miles de pesos (1993=100); el producto de Estados Unidos $\left(\mathrm{X}_{\mathrm{t}}\right)$ está en billones de dólares $(2000=100)$; el salario $\left(\mathrm{W}_{\mathrm{t}}\right)$ es el mínimo general promedio; el tipo de cambio nominal $\left(\mathrm{TC}_{\mathrm{t}}\right)$, el agregado monetario $\mathrm{M} 2\left(\mathrm{M}_{\mathrm{t}}\right)$, el índice nacional de precios al consumidor $\left(\mathrm{P}_{\mathrm{t}}\right)$, los precios de la energía $\left(\mathrm{PE}_{\mathrm{t}}\right)$ se obtuvieron del Banco de México; los precios relativos de la energía ( $\mathrm{PRE}_{\mathrm{t}}$ ) se calcularon de la relación $\left(\mathrm{PE}_{\mathrm{t}} / \mathrm{P}_{\mathrm{t}}\right)$. El producto de Estados Unidos $\left(\mathrm{X}_{\mathrm{t}}\right)$ se obtuvo del Banco Federal de la Reserva de Saint Louis. Las letras minúsculas representan el logaritmo natural de las series.

Las pruebas de raíces unitarias de Dickey Fuller Aumentada (ADF) (1981), Phillips Perron (PP) (1988) y Kwiatowsky et al. (KPSS) (1992) se sintetizan en el Cuadro 1. Los principales resultados de esas pruebas indican que la demanda de energía, el producto, los precios relativos, la inversión, el tipo de cambio real y el producto de Estados Unidos son estacionarios de orden uno, es decir son I(1), a 5\% de significancia. El índice de precios, el tipo de cambio nominal, el salario real y el agregado

$4 \quad$ Una descripción más detallada de la información se encuentra en el apéndice. 
Cuadro 1

Pruebas de raíces unitarias

\begin{tabular}{|c|c|c|c|c|c|c|c|c|}
\hline \multicolumn{2}{|c|}{ Variable } & \multicolumn{2}{|l|}{$A D F(4)$} & \multicolumn{3}{|c|}{$P P(3)$} & \multicolumn{2}{|c|}{$\operatorname{KPSS}(6)$} \\
\hline & $A$ & $B$ & C & A & $B$ & C & $\eta \mu$ & $\eta \tau$ \\
\hline$t_{t}$ & $-1.216(0)$ & $-4.393(0)$ & $1.562(2)$ & -1.227 & -4.065 & 4.072 & 0.638 & 0 . \\
\hline te & $-5.525(0)$ & $-2.279(1)$ & -1.489 (1) & -5.658 & -4.338 & -2.401 & 0.495 & 0.0 \\
\hline$y_{t}{ }^{t}$ & $-1.736(0)$ & $-2.915(0)$ & $3.054(1)$ & -1.782 & -2.664 & 5.190 & 0.668 & 0. \\
\hline$\Delta y_{t}$ & $-4.736(1)$ & $-4.303(0)$ & $2.613(0)$ & -4.713 & -4.309 & -2.405 & 0.383 & 0.0 \\
\hline & $-0.890(0)$ & $-0.219(0)$ & $-0.706(0)$ & -0.626 & -0.223 & -0.725 & 0.264 & 0.1 \\
\hline$\Delta$ pre $_{\mathrm{t}}$ & $-6.912(0)$ & $-6.225(0)$ & $-6.216(0)$ & -7.032 & -6.227 & -6.218 & 0.466 & 0.0 \\
\hline$i_{t}$ & $-2.600(0)$ & $-1.597(0)$ & $2.232(0)$ & -2.671 & -1.597 & 2.282 & 0.651 & 0 . \\
\hline$\Delta$ it & $-5.178(0)$ & $-5.216(0)$ & $-4.767(0)$ & -5.065 & -5.125 & -4.699 & 0.168 & 0.1 \\
\hline tcr $_{t}$ & $-3.070(0)$ & $-3.002(0)$ & $-0.360(2)$ & -3.075 & -3.020 & -0.337 & 0.147 & 0.0 \\
\hline$\Delta \mathrm{tcr}_{\mathrm{t}}$ & $-6.062(1)$ & $-6.143(1)$ & $-6.228(1)$ & -5.686 & -5.810 & -5.930 & 0.080 & 0.0 \\
\hline$x_{t}$ & $-2.504(1)$ & $0.256(0)$ & $9.768(0)$ & -2.196 & 0.272 & 10.043 & 0.692 & 0.1 \\
\hline$\Delta x_{t}$ & $-5.053(0)$ & $-5.050(0)$ & $-0.405(4)$ & -4.946 & -4.956 & -2.145 & 0.143 & 0.1 \\
\hline $\mathrm{p}_{\mathrm{t}}$ & $-2.186(3)$ & $-0.903(1)$ & $-1.351(3)$ & -1.662 & -0.258 & -0.278 & 0.652 & 0.0 \\
\hline$\Delta \mathrm{p}_{\mathrm{t}}$ & $-2.261(0)$ & $-2.325(0)$ & $-1.558(0)$ & -2.104 & -2.216 & -1.367 & 0.148 & 0.1 \\
\hline $\mathrm{s}_{\mathrm{t}}$ & $-2.297(3)$ & $-0.673(1)$ & $-1.408(3)$ & -1.882 & -0.195 & -1.193 & 0.635 & 0.09 \\
\hline$\Delta \mathrm{s}_{\mathrm{t}}$ & $-2.829(0)$ & $-2.904(0)$ & $-1.506(2)$ & -2.718 & -2.807 & -2.221 & 0.146 & 0.13 \\
\hline$w_{t}$ & $-2.462(2)$ & $-0.786(1)$ & $-1.459(2)$ & -1.549 & -0.411 & -0.663 & 0.657 & $0.0 s$ \\
\hline$\Delta \mathrm{w}_{\mathrm{t}}$ & $-2.330(0)$ & $-2.362(0)$ & $-1.046(1)$ & -2.337 & -2.394 & -1.527 & 0.140 & 0.13 \\
\hline$m_{t}$ & $-1.835(1)$ & $-0.611(1)$ & 1.160 & -1.707 & -0.036 & 4.730 & 0.668 & 0.10 \\
\hline$\Delta \mathrm{m}_{\mathrm{t}}$ & $-2.380(0)$ & - $2.467(0)$ & $-1.277(0)$ & -2.202 & -2.329 & -1.044 & 0.154 & 0.15 \\
\hline
\end{tabular}

Notas: los valores en negritas representan el rechazo de la hipóstesis nula a 5\%. Los valores críticos a 5\% de significancia para la prueba Dickey-Fuller Aumentada y Phillips-Perron para una muestra de $\mathrm{T}=100$ son -3.45 , incluyendo constante y tendencia (modelo A), -2.89 incluyendo constante (modelo B) y -1.95 sin constante y tendencia (modelo C) (Maddala y Kim, 1998:64). $\eta$ y $\eta \tau$ representan los estadísticos de la prueba KPSS donde la hipótesis nula considera que la serie es estacionaria en nivel o alrededor de una tendencia determinística, respectivamente. Los valores críticos a 5\% de ambas pruebas son 0.463 y 0.146 , respectivamente (Kwiatkowski et al., 1992:166). Las letras en minúsculas representan los valores en logaritmos naturales. Los valores entre paréntesis son el número de rezagos. Periodo 1965-2003.

monetario M2 son I(1), pero a 10\% de significancia en la mayoría de las pruebas. De ese modo, los resultados de las pruebas de raíces unitarias sugieren el uso de un método de cointegración, tal como el de Johansen (1988).

El procedimiento de Johansen (1988), corregido por la posible presencia de cambio estructural, con base en el método propuesto por Hansen y Johansen (1993), indica que existe al menos un vector de cointegración atendiendo a la prueba de la traza para cada una de las ecuaciones consideradas (cuadros 2, 3 y 4). Asimismo, las pruebas de estabilidad estructural de los vectores de cointegración representadas por los modelos $\mathrm{R} \mathrm{y} \mathrm{Z}$ (véanse en el anexo las gráficas $1 \mathrm{~A}, 2 \mathrm{~A}, 3 \mathrm{~A}$ ) no rechazan la hipótesis nula para los tres vectores de cointegración. Ello no obstante que el 
Cuadro 2

Prueba de cointegración basada en el procedimiento de Johansen (1988) y Hansen y Johasen (1993)

\begin{tabular}{ccccc}
\hline \multicolumn{5}{c}{ et $=\boldsymbol{\beta}_{\mathbf{1}}{ }^{*} \mathbf{y}_{\mathbf{t}}+\boldsymbol{\beta}_{\mathbf{2}}{ }^{*} \mathbf{p r e} \mathbf{t}_{\mathbf{t}}$} \\
\hline$H_{0}$ & Constante & Tendencia & Traza & $95 \%$ \\
\hline $\mathrm{r}=0$ & 0 & 0 & $55.82^{* * *}$ & 24.31 \\
$\mathrm{r} \leq 1$ & 0 & 0 & 7.52 & 12.53 \\
$\mathrm{r} \leq 2$ & 0 & 0 & 0.58 & 3.84 \\
\hline
\end{tabular}

Notas: $(*)$ Rechazo a 5\% de nivel de significancia. $(* *)$ Rechazo a $1 \%$ de significancia. Traza $=$ prueba de la traza. $r=$ número de vectores de cointegración. Número de rezagos en el VAR $=1$.

Cuadro 3

Estadísticos de cointegración basados en el procedimiento de Johansen (1988) y Hansen y Johasen (1993)

\begin{tabular}{ccccc}
\hline \multicolumn{5}{c}{$\mathbf{y}_{\mathbf{t}}=\boldsymbol{\beta}_{\mathbf{1}} *_{\mathbf{i}}+\boldsymbol{\beta}_{\mathbf{2}} * \mathbf{t c r}_{\mathbf{t}}+\boldsymbol{\beta}_{\mathbf{3}} * \mathbf{x}_{\mathbf{t}}-\boldsymbol{\beta}_{\mathbf{4}} * \mathbf{p r e}_{\mathbf{t}}$} \\
\hline$H_{0}$ & Constante & Tendencia & Traza & $95 \%$ \\
\hline $\mathrm{r}=0$ & ab0 & 0 & $198.94 * *$ & 66.33 \\
$\mathrm{r} \leq 1$ & $\mathrm{ab0}$ & 0 & $102.11 * *$ & 42.41 \\
$\mathrm{r} \leq 2$ & $\mathrm{ab0}$ & 0 & $57.60 * *$ & 31.93 \\
$\mathrm{r} \leq 3$ & $\mathrm{ab0}$ & 0 & $24.24 *$ & 18.32 \\
$\mathrm{r} \leq 4$ & $\mathrm{ab0}$ & 0 & 4.70 & 8.41 \\
\hline
\end{tabular}

Notas: $(*)$ Rechazo a $5 \%$ de nivel de significancia, $(* *)$ Rechazo a $1 \%$ de significancia. Traza = prueba de la traza. $r=$ número de vectores de cointegración. Número de rezagos en el vaR $=1$. El var incluye una variable dummy de cambio de pulso (1995) unrestricted. Los valores críticos del estadístico de la traza fueron calculados con el programa DisCo con las siguientes opciones: simulación para 41 observaciones, 20,000 iteraciones y una variables restricted.

Cuadro 4

Prueba de cointegración basada en el procedimiento de Johansen (1988) y de Hansen y Johasen (1993)

\begin{tabular}{ccccc}
\hline \multicolumn{5}{c}{$\mathbf{p}_{\mathbf{t}}=\boldsymbol{\beta}_{\mathbf{1}}{ }^{*} \mathbf{t c}_{\mathbf{t}}+\boldsymbol{\beta}_{\mathbf{2}}{ }^{*} \mathbf{w}_{\mathbf{t}}+\boldsymbol{\beta}_{\mathbf{3}}{ }^{*} \mathbf{m} \mathbf{2}_{\mathbf{t}}+\boldsymbol{\beta}_{\mathbf{4}}{ }^{*} \mathbf{p r e}$} \\
\hline$H_{0}$ & Constante & Tendencia & Traza & $95 \%$ \\
\hline $\mathrm{r}=0$ & 0 & 0 & $127.94 * *$ & 68.52 \\
$\mathrm{r} \leq 1$ & 0 & 0 & $62.14^{* *}$ & 47.21 \\
$\mathrm{r} \leq 2$ & 0 & 0 & $30.62^{*}$ & 29.68 \\
$\mathrm{r} \leq 3$ & 0 & 0 & 7.89 & 15.41 \\
$\mathrm{r} \leq 4$ & 0 & 0 & 0.17 & 3.76 \\
\hline
\end{tabular}

Notas: (*) Rechazo a 5\% de nivel de significancia (**) Rechazo a 1\% de significancia. Traza = prueba de la traza. $r=$ número de vectores de cointegración. Número de rezagos en el VAR $=4$. 
tercer vector presenta problemas con el modelo Z, pero en el caso de que los resultados entre el modelo $\mathrm{Z}$ y el R sean contradictorios se opta por el segundo estadístico (Hansen y Johansen, 1993). Esto es, el rango y el espacio de cointegración se mantiene estable a lo largo del periodo de la submuestra (Mosconi, 1998), por lo que no se registró cambio estructural.

Normalizando a los vectores de cointegración de acuerdo con la especificación sugerida en las ecuaciones 1, 2 y 3 , se observa que se obtienen parámetros razonables y consistentes con la teoría económica. Esto es, la ecuación (8) indica que la elasticidad ingreso de la demanda de energía es de 0.60 y la elasticidad precio es -0.19. Ello indica que el consumo de energía está altamente correlacionado con la actividad económica, y que los precios relativos de la energía tienen una relación inversa a su consumo; es interesante notar que el coeficiente es relativamente alto en relación con otros estudios disponibles (por ejemplo, Galindo y Sánchez, 2005). Sin embargo, en años recientes los precios de la energía han presentado fuertes incrementos y esto, evidentemente, ha repercutido en su demanda.

$$
e_{t}=0.60 * y_{t}-0.19 * \text { pre }_{t}
$$

Por su parte, la ecuación (9) indica que el producto responde positivamente a la inversión, al producto de Estados Unidos y al tipo de cambio real; y negativamente a un aumento de los precios relativos de la energía. Esta evidencia es consistente con trabajos previos, tales como Jorgenson y Wilcoxen (1998) para Estados Unidos.

$$
\Delta \mathrm{y}_{\mathrm{t}}=0.73 * \mathrm{i}_{\mathrm{t}}+0.24 * \mathrm{tcr}_{\mathrm{t}}+0.21 * \mathrm{x}_{\mathrm{t}}-0.10 * \text { pre }_{\mathrm{t}}+2.89
$$

Finalmente, el nivel de precios se ajusta positivamente al aumento en el tipo de cambio nominal, los salarios, el agregado monetario M2 y los precios relativos de la energía. Esta evidencia es consistente con las hipótesis de formación de precios de costos normales (Ros, 1981) o de sensibilidad (o pass through) de algunos precios clave a la inflación (Gil Díaz y Ramos Tercero, 1988 y Schmidt-Hebbel y Werner, 2002) junto a un efecto de propagación o de validación de la inflación asociado al comportamiento del agregado monetario (Gómez, 1975).

$$
\Delta \mathrm{p}_{\mathrm{t}}=0.41 * \mathrm{tc}_{\mathrm{t}}+0.63 * \mathrm{w}_{\mathrm{t}}+0.10 * \mathrm{~m} 2_{\mathrm{t}}+0.12 * \text { pre }_{\mathrm{t}}
$$

Los vectores de cointegración obtenidos pueden utilizarse entonces de acuerdo con el teorema de representación de Engle y Granger (1987), para obtener los 
modelos econométricos finales. Estos modelos econométricos finales (ecuaciones 11,12 y 13) muestran una alta capacidad de reproducir el comportamiento histórico de los datos, como se observa en los coeficientes de determinación y las gráficas 1, 2 y 3 . Asimismo, no existen signos de autocorrelación o heteroscedasticidad en los residuales y no se rechaza la hipótesis de normalidad en los errores. De esa forma, esos tres modelos pueden considerarse una aproximación adecuada al proceso generador de información (Spanos, 1986).

Así, las estimaciones del modelo econométrico final para la ecuación 11 son:

$$
\begin{gathered}
\Delta \mathrm{de}_{\mathrm{t}}=0.68 * \Delta \mathrm{y}_{\mathrm{t}}-0.04 * \mathrm{ECM}_{\mathrm{t}-1}-0.06 * \mathrm{D} 99+0.05 * \mathrm{D} 04 \\
(7.51) \quad(-3.29)
\end{gathered}
$$

$\mathrm{R}^{2}=0.79$

$\mathrm{J}-\mathrm{B}=0.57(0.75)$

LM (2): $F=0.68(0.51)$

ARCH (2):F $=0.38(0.54)$

Error cuadrático medio $=0.02$

Y para la ecuación 12 se obtuvieron las siguientes:

$$
\begin{aligned}
& \Delta \mathrm{y}_{\mathrm{t}}=0.26 * \Delta \mathrm{f}_{\mathrm{t}}+0.14 * \Delta \mathrm{x}_{\mathrm{t}}-0.03 * \Delta \mathrm{pre}_{\mathrm{t}-2}-0.15 * \mathrm{ECM}_{\mathrm{t}-1}-0.02 * \Delta 86 \\
& \begin{array}{llll}
(22.15) & (2.32) \quad(-1.95) & (-14.01) & (-2.49)
\end{array}
\end{aligned}
$$

$\mathrm{R}^{2}=0.95$

$\mathrm{J}-\mathrm{B}=0.84(0.66)$

LM (2): $F=0.61(0.55)$

$\mathrm{ARCH}(2): \mathrm{F}=1.70(0.20)$

Error cuadrático medio $=0.007$

Finalmente, para la ecuación 13, de precios, las estimaciones son:

$$
\begin{array}{ccc}
\Delta \mathrm{p}_{\mathrm{t}}=0.55 * \Delta \mathrm{tc}_{\mathrm{t}}+0.54 * \Delta \mathrm{w}_{\mathrm{t}}-0.16 * \mathrm{ECM}_{\mathrm{t}-1}-0.15 * \mathrm{D} 73-0.14 * \mathrm{D} 88 \\
(10.14) & (7.90) & (-2.29)
\end{array}
$$

$\mathrm{R}^{2}=0.92$

$\mathrm{J}-\mathrm{B}=0.07(0.96)$

LM (2): $\mathrm{F}=1.48(0.24)$

$\mathrm{ARCH}(2): \mathrm{F}=0.27(0.75)$

Error cuadrático medio $=0.04$ 


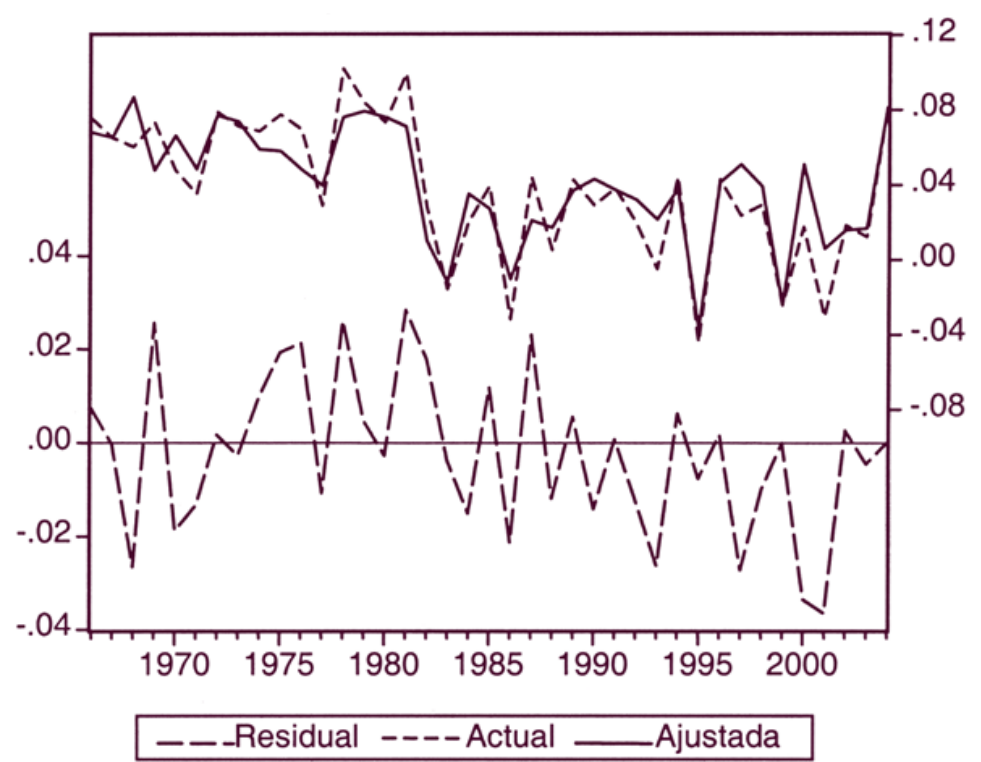

Gráfica 1. Modelo econométrico final de la demanda de energía.

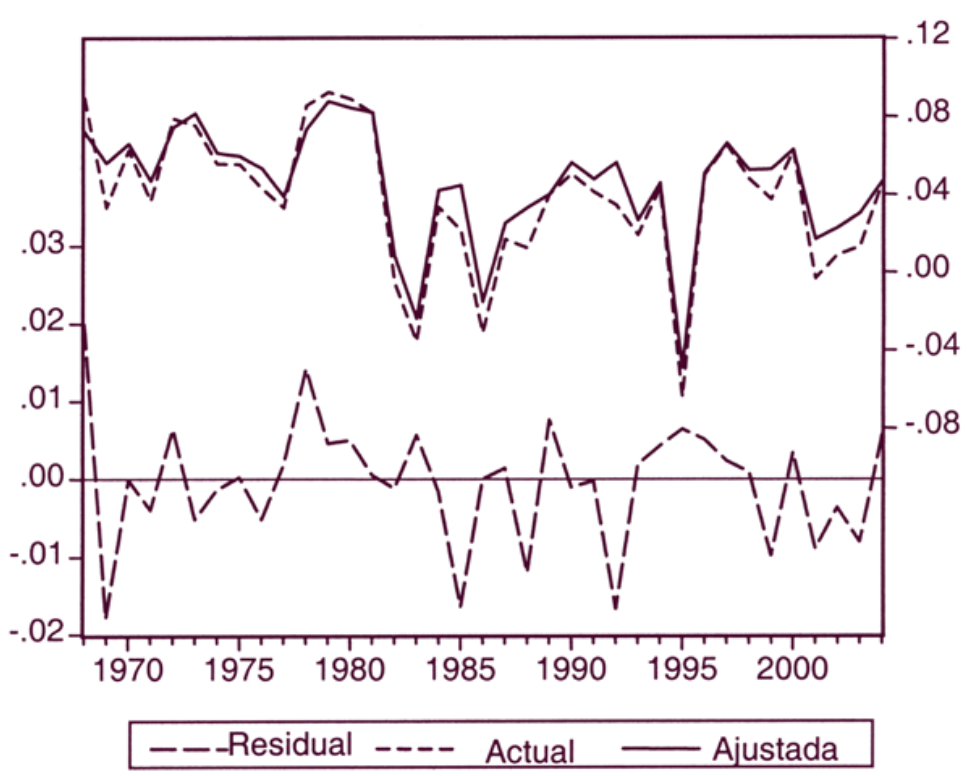

Gráfica 2. Modelo econométrico final del producto. 


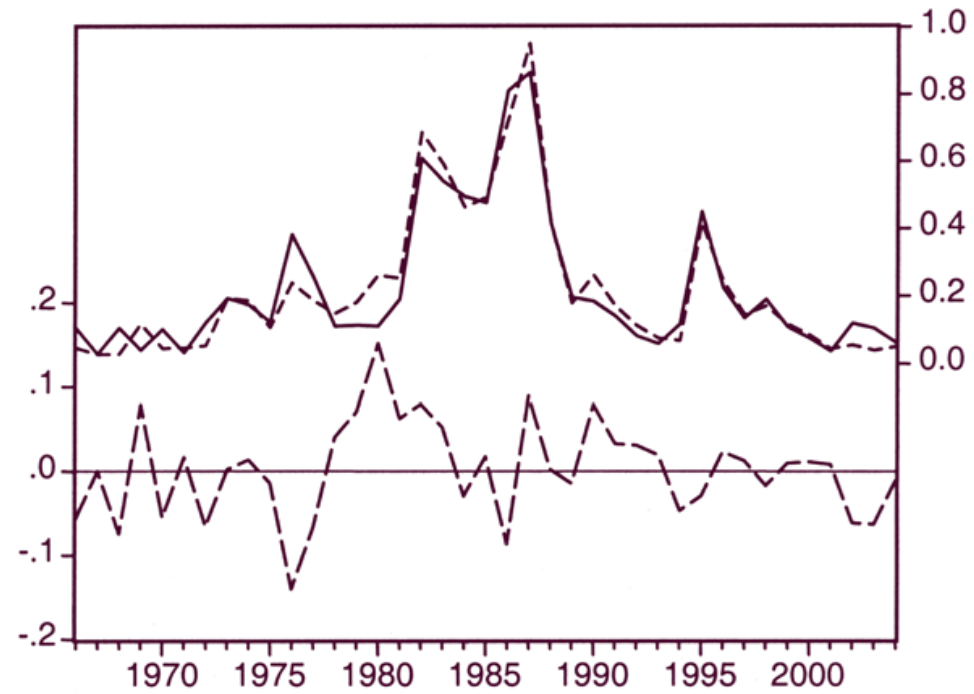

- - Residual --- Actual — Ajustada

Gráfica 3. Modelo econométrico final de precios.

Cuadro 5

Simulaciones base

\begin{tabular}{|c|c|c|c|c|c|c|}
\hline \multirow[b]{2}{*}{$A \tilde{n} o$} & \multicolumn{2}{|c|}{ Escenario 1} & \multicolumn{2}{|c|}{ Escenario 2} & \multicolumn{2}{|c|}{ Escenario 3} \\
\hline & $\Delta E_{t}$ & $\Delta Y_{t}$ & $\Delta E_{t}$ & $\Delta Y_{t}$ & $\Delta E_{t}$ & $\Delta Y_{t}$ \\
\hline 2005 & 2.23 & 2.5 & 2.91 & 3.5 & 3.60 & 4.5 \\
\hline 2006 & 2.21 & 2.5 & 2.89 & 3.5 & 3.57 & 4.5 \\
\hline 2007 & 2.18 & 2.5 & 2.86 & 3.5 & 3.54 & 4.5 \\
\hline 2008 & 2.16 & 2.5 & 2.83 & 3.5 & 3.51 & 4.5 \\
\hline 2009 & 2.14 & 2.5 & 2.81 & 3.5 & 3.48 & 4.5 \\
\hline 2010 & 2.12 & 2.5 & 2.78 & 3.5 & 3.45 & 4.5 \\
\hline 2011 & 2.09 & 2.5 & 2.76 & 3.5 & 3.42 & 4.5 \\
\hline 2012 & 2.07 & 2.5 & 2.73 & 3.5 & 3.40 & 4.5 \\
\hline 2013 & 2.05 & 2.5 & 2.71 & 3.5 & 3.37 & 4.5 \\
\hline 2014 & 2.03 & 2.5 & 2.69 & 3.5 & 3.35 & 4.5 \\
\hline 2015 & 2.02 & 2.5 & 2.67 & 3.5 & 3.33 & 4.5 \\
\hline
\end{tabular}

Las ecuaciones econométricas obtenidas permiten, entonces, realizar diversas simulaciones y proyecciones sobre los efectos macroeconómicos de los cambios en los precios de la energía y los posibles escenarios base entre crecimiento económico y consumo de energía. En efecto, el Cuadro 5 presenta el aumento del consumo de 


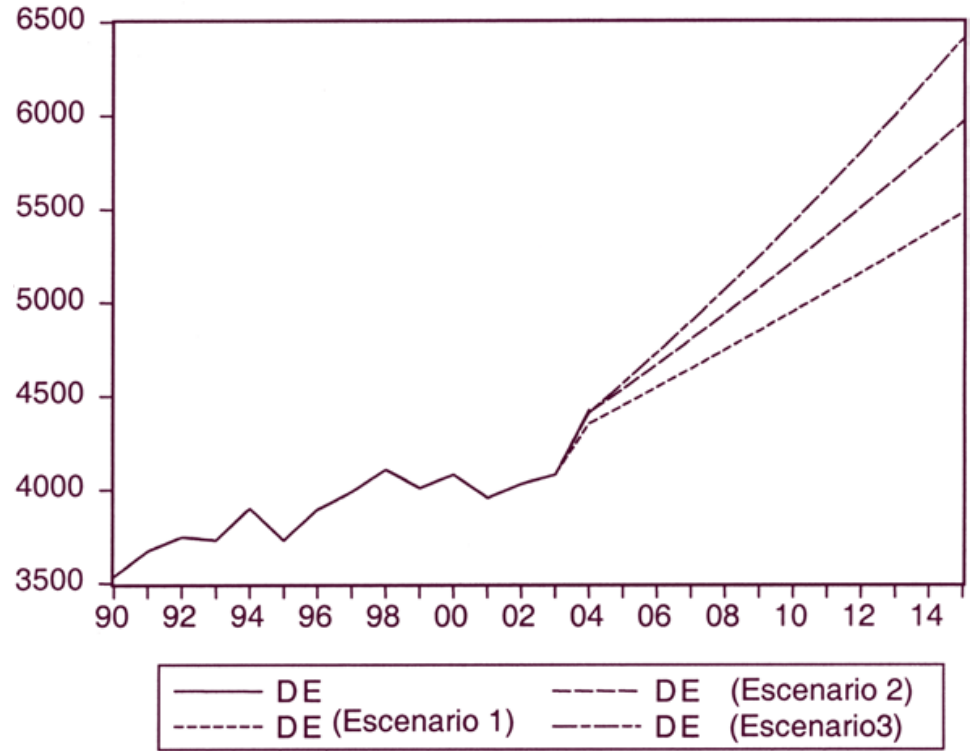

Gráfica 4. Pronóstico de la demanda de energía para los diferentes escenarios.

energía ante distintas alternativas de ritmo de crecimiento económico, ${ }^{5}$ bajo el supuesto de que los precios relativos de la energía igualmente sean constantes. A pesar de que las tasas de crecimiento del producto se mantienen constantes en cada escenario, se puede observar que el aumento del consumo de energía presentan una ligera tendencia a la baja asociado al impacto de los precios. A pesar de ello, las altas tasas de crecimiento de consumo energético representan un ritmo de crecimiento insostenible en el largo plazo dentro de un contexto de un desarrollo económico sustentable (véase Gráfica 4), bajo los tres escenarios modelados.

Asimismo, se realizaron pronósticos simultáneos con base en el algoritmo de Gauss Seidel de los tres modelos econométricos finales. Esos resultados muestran que existe una fuerte interdependencia entre el producto, los precios y el consumo de energía. En este contexto, las simulaciones realizadas considerando diversos precios de la energía se sintetizan en el Cuadro $6,{ }^{6}$ en el cual se observa que a pesar de fuertes incrementos en los precios relativos de la energía las trayectorias de crecimiento económico y consumo de energía no llegan a desacoplarse, aunque las tasas de crecimiento disminuyen.

5 Se supone un ritmo de crecimiento constante en cada alternativa.

6 Los supuestos para las demás variables se tomaron de los criterios generales de política económica para 2006, y se mantuvieron constantes para los demás años. 
Cuadro 6

Simulaciones de tasas de crecimiento de producto, precios y demanda de energía 2005-2015

\begin{tabular}{lccccccccccccc}
\hline Año & \multicolumn{4}{c}{$\Delta$ pre $_{t}=0 \%$} & \multicolumn{4}{c}{$\Delta$ spre $_{t}=5 \%$} & \multicolumn{4}{c}{$\Delta$ spe $_{t}=10 \%$} & \multicolumn{4}{c}{$\Delta p r e_{t}=25 \%$} \\
& $\Delta Y_{t}$ & $\Delta P_{t}$ & $\Delta E_{t}$ & $\Delta Y_{t}$ & $\Delta P_{t}$ & $\Delta E_{t}$ & $\Delta Y_{t}$ & $\Delta P_{t}$ & $\Delta E_{t}$ & $\Delta Y_{t}$ & $\Delta P_{t}$ & $\Delta E_{t}$ \\
\hline & & & & & & & & & & & & \\
2005 & 3.43 & 3.22 & 2.54 & 3.43 & 3.22 & 2.54 & 3.43 & 3.22 & 2.54 & 3.43 & 3.22 & 2.54 \\
2006 & 3.19 & 3.70 & 2.34 & 3.11 & 3.80 & 2.26 & 3.04 & 3.88 & 2.18 & 2.85 & 4.13 & 1.97 \\
2007 & 3.58 & 3.91 & 2.58 & 3.29 & 4.09 & 2.32 & 3.01 & 4.25 & 2.07 & 2.19 & 4.70 & 1.35 \\
2008 & 3.74 & 4.16 & 2.66 & 3.42 & 4.40 & 2.35 & 3.11 & 4.63 & 2.05 & 2.22 & 5.25 & 1.20 \\
2009 & 3.89 & 3.89 & 2.73 & 3.54 & 4.18 & 2.37 & 3.20 & 4.46 & 2.03 & 2.24 & 5.24 & 1.06 \\
2010 & 4.01 & 3.65 & 2.79 & 3.63 & 4.00 & 2.39 & 3.27 & 4.33 & 2.00 & 2.26 & 5.23 & 0.93 \\
2011 & 4.11 & 3.46 & 2.83 & 3.72 & 3.84 & 2.39 & 3.34 & 4.21 & 1.97 & 2.28 & 5.22 & 0.79 \\
2012 & 4.20 & 3.30 & 2.86 & 3.79 & 3.71 & 2.39 & 3.39 & 4.11 & 1.93 & 2.30 & 5.21 & 0.66 \\
2013 & 4.28 & 3.16 & 2.89 & 3.85 & 3.60 & 2.38 & 3.44 & 4.03 & 1.89 & 2.31 & 5.20 & 0.53 \\
2014 & 4.34 & 3.04 & 2.91 & 3.90 & 3.51 & 2.36 & 3.48 & 3.96 & 1.84 & 2.32 & 5.19 & 0.41 \\
2015 & 4.40 & 2.94 & 2.92 & 3.95 & 3.43 & 2.35 & 3.52 & 3.90 & 1.80 & 2.33 & 5.19 & 0.29 \\
\hline
\end{tabular}

Los efectos de los aumentos de precios de la energía también tienen consecuencias en el comportamiento del producto y los precios. Por ejemplo, para 2006, ante un shock de $5 \%$ en los precios relativos de la energía, se produjo una disminución del consumo de ésta de $0.08 \%$, así como en un aumento de la tasa de inflación de $0.09 \%$ y una disminución del producto de $0.07 \%$. Esas variaciones se van incrementando con el tiempo, así para 2015 la demanda de energía disminuiría $0.57 \%$ para el mismo incremento de los precios relativos. Asimismo, las demás simulaciones realizadas muestran también la interdependencia de las variables consideradas.

\section{Conclusiones y comentarios generales}

Los resultados obtenidos en este trabajo indican que es posible establecer relaciones estables de largo plazo, representadas por los vectores de cointegración y que pueden interpretarse como ecuaciones de demanda de energía, de producto y de precios. Así, la demanda de energía responde positivamente a la trayectoria del ingreso y negativamente al comportamiento de los precios relativos. En particular, se observa una elasticidad ingreso de 0.60 y una elasticidad precio de - 0.19 . Asimismo, el producto responde negativamente a un aumento de los precios de la energía. Ello refleja, desde el punto de vista de una función de producción, mayores costos, lo que es consistente con la evidencia internacional al respecto (Jorgenson, 1998). Finalmente, se observa que el nivel de precios responde positivamente a un alza de los precios relativos de la energía. Estos vectores de cointegración permiten cons- 
truir modelos econométricos finales con las propiedades estadísticas adecuadas y con una alta capacidad para simular y proyectar el comportamiento de las variables endógenas seleccionadas.

Las simulaciones y proyecciones realizadas con estos modelos indican claramente que existe una fuerte dependencia y asociación entre el consumo de energía y el producto, que los movimientos en los precios en un rango razonable no parecen poder compensar. Además, se observa que cambios bruscos e importantes de precios generan efectos negativos en el producto, la demanda de energía y la inflación.

En ese sentido, es de esperarse que una recuperación del crecimiento económico en México venga acompañada de un mayor consumo de energía. Este aumento del consumo energético difícilmente se verá frenado por un incremento de los precios de la energía, sin embargo, los cambios tecnológicos y las fuentes de energía alternativa podrían hacer más eficiente su uso y la generación de la misma. Por lo tanto, ante una política de aumento de precios para controlar el consumo de energía, sería una medida que tendrá también consecuencias negativas en la evolución del producto y los precios generales de la economía. Estos resultados muestran las posibilidades y limitaciones en el uso de los precios de la energía como instrumento de política pública, pero también sugieren las dificultades para transitar a un proceso de crecimiento económico sustentable. Así pues, una evaluación económica general de los precios de la energía debe incluir sus consecuencias sobre el desempeño general de la economía y no debe realizarse sólo atendiendo a sus consecuencias más inmediatas.

Debido a la escasa eficiencia de los precios de la energía como instrumento para controlar el uso de los energéticos, se debe entonces pensar en políticas dirigidas a mejorar la eficiencia energética, el cambio tecnológico y el desarrollo de fuentes alternativas de energía. Si bien en conjunto el efecto de los precios relativos no es muy significativo en la demanda de energía, no obstante ello, es necesario revisar los diferentes energéticos y los impactos de precios diferenciados de forma que sean consistentes con una trayectoria de crecimiento sustentable.

\section{Bibliografía}

Aguayo F. y K. P. Gallagher, "Economic reform, energy, and development: the case of
Mexican manufacturing", Energy policy, vol. 33, núm. 7, 2005, pp. 829-837. 
Baffes, J. y J.C. Le Valle, "Unit roots versus trend stationary in growth rate estimation", Applied Economics Letters, vol. 10, núm. 1, 2003, pp. 9-14.

Bernanke, Mishkin et al., "Inflation targeting a new framework for monetary policy?", NBER Working Paper, núm. W5893, 1997.

Böhringer, C., "The synthesis of bottom-up and top-down in energy policy modeling", Energy Economics, núm 20, 1998, pp. 233-248.

Dickey, D.A. y W.A Fuller., "Likelihood ratio statistics for autoregressive time series with a unit root", Econometrica, núm 49, 1981, pp. 1057-1072.

Engle, R.F. y C.W.J. Granger, "Cointegration and error correction: representation, estimation and testing", Econometrica, núm. 55, 1987, pp. 251-276.

Garcés, D.D., "La relación de largo plazo del PIB mexicano y de sus componentes con la actividad económica en los Estados Unidos y con el tipo de cambio real", Banco de México, Documento de investigación, núm. 4, México, 2003.

- "Determinación del nivel de precios y la dinámica inflacionaria en México", Gaceta de Economía ITAM, núm. 7 Especial, México, 2002.

Galindo, L.M. y L. Sánchez, "El consumo de energía y la economía mexicana: un análisis comparativo con VAR", Economía mexicana, vol. XIV, núm. 2, México, Nueva época, 2004.

Galindo, L.M., "Short and long-run demand for energy in Mexico: A cointegration approach", Energy Policy, vol. 33, núm.9, 2005.

Gil Díaz, Francisco y Raúl Ramos, "Lecciones desde México", en M. Bruno y otros (compiladores), Inflación y Estabilización, Lecturas de El Trimestre Económico, núm. 62, México, FCE, 1998.

Graham, P.W. y D.J. Williams, "Optimal technological choices in meeting Australian energy policy goals", Energy Economics, vol. 25, núm. 6, 2003, pp. 691-712.

Gómez, O., "La demanda de dinero en México", Cincuenta Años de Banca Central, México, FCE, 1975.

Guerrero, C.L. "Determinantes del crecimiento económico en México, 1929-2003, una perspectiva postkeynesiana", serie de documentos de trabajo EGAP, EC 04 09, 2004.

Gutés, M.C., "The concept of weak sustainability", Ecological Economics, núm. 17, 1996, pp. 147-156.
Hannan E. J. y B. G. Quinn, "The determination of the order of an autoregression", Journal of the Royal Statistical Society, 1979, vol. 41, núm. 2, pp. 190-195.

Hansen, H. y S. Johansen, Recursive Estimation in Cointegrated VAR Models, University of Copenhagen, Institute of Mathematical Statistics, pre-print 93-1, 1993.

Hartwick, J., "Intergenerational equity and investing of rents from exhaustible resources", American Economic Review, vol. 66, 1977, pp. 972-974.

Ho, M.S. y D.W. Jorgenson "Stabilization of carbon emission and international competitiveness of U.S. industries", en Growth. Econometric General Equilibrium Modeling, vol. 2, Cambridge, The мIт Press, D.W. Jorgenson ,1998, pp. 373-412.

Howarth, R.B., L. Schipper y B. Anderson, "The structure and trends and intensity of energy use: trends in five OECD nations", The Energy Journal, vol.14, núm. 2, 1993, pp. 27-44.

Johansen, S, "Statistical analysis of cointegration vectors", Journal of Economic Dynamics and Control, 12, 1988, pp. 231-254.

___ Likelihood Based Inference on Cointegration in the Vector Autoregressive Model, Oxford University Press, Oxford, 1995.

Johansen, S. y B.G. Nielsen, "Asymptotics for cointegration ranks test in the presence of intervention dummies", 1993, Manual for the Simulation Program DisCo, http:// www.nuff.ox.ac.uk/users/nielsen/disco.html.

Jorgenson, D.W., "Econometric methods for applied general equilibrium analysis", en H.E. Scarf y J.B. Shoven (editores), Applied General Equilibrium Analysis, Cambridge 1984, pp. 139-203.

_, "Growth: Energy", The Environmental and Economics Growth, vol.2, MIT Press. 1998.

Jorgenson, D.W. y P.J. Wilcoxen, "Energy, the environment and economic growth", en A. Knees y J. Sweeney (editores), Handbook of Natural Resource and Energy Economics, vol. 3, 1993a, pp.1267-1349.

, "Fundamental tax reforms and energy markets", en D.W. Jorgenson (editor), Growth. Econometric general Equilibrium Modeling, vol. 2, Cambridge, , 1998, pp. 413-441.

King, R.G. y R. Levine, "Finance and growth: Schumpeter might be right", Quarterly 
Journal of Economics, vol. 108, 1994, pp. 717-737.

Kwiatkowski, D.; P.C.B. Phillips, P. Schmidt y Y. Shin, "Testing the null hypothesis of stationary against the alternative of a unit root", Journal of Econometrics, vol. 1, 1992, pp. 159-178.

Leybourne, S. y P. Newbold, "Behavior of Dickey-Fuller t-test when there is a break under the alternative hypothesis", Econometric Theory, vol. 16 núm. 5, octubre 2000, pp. 779-789.

Mabey, N.; S. Hall, C. Smith, y S. Gupta, Argument in the Greenhouse. The International Economics of Controlling Global Warming, Routledge, 1997.

Maddala, G.S. y I. Kim, Unit roots, cointegration and structural change, Cambridge University Press, 1998.

Mosconi, R., MALCOLM (Maximum Likelihood Cointegration Analysis in RATS), Cafoscarina, Venice, 1998.

Nachane, D. M.; R.M. Nadkarni, y A.V. Karnik, "Cointegration and causality testing of the energy-GDP relationship: a cross-country study", Applied Economics, vol. 20, 1998, pp. 1511-1531.

$\mathrm{Ng}$, S. y P. Perron, "Unit root tests in ARMA models with data depend methods for the selection of the truncation lag", Journal of the American Statistical Association, vol. 90, 1995, pp. 268-281.

Obstfeld, M. y K. Rogoff, Foundations of International macroeconomics, MIT press. 1999.

Pearce, D.W. y G. Atkinson, "Measuring sustainable development", The Handbook of Environmental Economics, Oxford, D.W. Bromley Blackwell, 1995.

Pearce, D.W.; E.B. Barbier y A. Markandya, Sustainable Development: Economics and Environment in the Third World, Edward Elgar Publishers, Aldershot and Vermont, 1990.

Phillips, P.C.B. y P. Perron, "Testing for unit roots in time series regression", Biometrika, vol. 75, 1988, pp.335-346.

Pindyck, R.S., The Structure of World Energy Demand, Cambridge, The MIT Press, 1979.

Ros J., Development Theory \& the Economics of Growth, The University of Michigan Press, 2000.

Ros J., "La inflación: la experiencia de la década de los setenta", en R. Cordera (editor), "Desarrollo y Crisis de la Economía Mexicana", Trimestre Económico, núm. 39, FCE, 1981.

Sala-i-Marti, X., "The classical approach to convergence analysis", Economic Journal, vol. 106, julio 1996, pp.1019-1036.

Schmidt-Hebbel K. y A. Werner, "Inflation Targeting in Brazil, Chile, and Mexico: Performance, Credibility, and the Exchange Rate." Central Bank of Chile Working Papers, núm. 171, 2002.

Solow, R.M., "On the intergenerational allocation of natural resources", Scandinavian Journal of Economics, vol. 88, 1986, pp. 141-149.

Spanos, A., Statistical Foundations of Econometric Modeling, Cambridge University Press, 1986. 
Cuadro A.1

Pruebas de incorrecta especificación sobre el procedimiento de Johansen de las ecuaciones

\begin{tabular}{llllllll}
\hline & \multicolumn{2}{c}{$L M(2)$} & \multicolumn{2}{c}{ ARCH(2) } & \multicolumn{3}{c}{$J B$} \\
\hline $\mathrm{de}_{\mathrm{t}}$ & 0.694 & {$[0.506]$} & 0.377 & {$[0.689]$} & 0.486 & {$[0.784]$} \\
$\mathrm{y}_{\mathrm{t}}$ & 0.664 & {$[0.512]$} & 0.022 & {$[0.979]$} & 3.672 & {$[0.159]$} \\
$\mathrm{pre}_{\mathrm{t}}$ & 0.870 & {$[0.428]$} & 1.462 & {$[0.247]$} & 3.840 & {$[0.147]$} \\
& & & & & & & \\
$\mathrm{y}_{\mathrm{t}}$ & 1.992 & {$[0.154]$} & 0.520 & {$[0.600]$} & 0.270 & {$[0.873]$} \\
$\mathrm{i}_{\mathrm{t}}$ & 2.797 & {$[0.077]$} & 1.602 & {$[0.219]$} & 1.057 & {$[0.589]$} \\
$\mathrm{tcr}_{\mathrm{t}}$ & 3.125 & {$[0.058]$} & 1.008 & {$[0.378]$} & 4.220 & {$[0.121]$} \\
$\mathrm{x}_{\mathrm{t}}$ & 1.086 & {$[0.350]$} & 0.477 & {$[0.625]$} & 4.961 & {$[0.084]$} \\
$\mathrm{pre}_{\mathrm{t}}$ & 0.100 & {$[0.905]$} & 0.134 & {$[0.875]$} & 6.213 & {$[0.045]^{*}$} \\
& & & & & & \\
$\mathrm{p}_{\mathrm{t}}$ & 0.035 & {$[0.854]$} & 0.143 & {$[0.867]$} & 2.467 & {$[0.291]$} \\
$\mathrm{tc}_{\mathrm{t}}$ & 0.442 & {$[0.517]$} & 0.107 & {$[0.899]$} & 3.092 & {$[0.213]$} \\
$\mathrm{w}_{\mathrm{t}}$ & 1.916 & {$[0.188]$} & 0.079 & {$[0.925]$} & 1.911 & {$[0.385]$} \\
$\mathrm{m}_{\mathrm{t}}$ & 0.993 & {$[0.336]$} & 0.018 & {$[0.983]$} & 3.475 & {$[0.176]$} \\
$\mathrm{pre}_{\mathrm{t}}$ & 0.019 & {$[0.895]$} & 0.106 & {$[0.900]$} & 4.094 & {$[0.129]$} \\
\hline
\end{tabular}

Notas: $(*)$ indica rechazo de la hipótesis nula. $\mathrm{LM}=$ prueba para autocorrelación, $\mathrm{ARCH}=$ prueba para heterocedasticidad y JB = prueba para normalidad. Para el VAR de EQ1 LM: $\mathrm{F}(2,34)$ y ARCH F(2,32), para EQ2 LM: $\mathrm{F}(2,30)$ y ARCH: $\mathrm{F}(2,28)$, y para EQ3 LM: $\mathrm{F}(2,14)$ y ARCH $\mathrm{F}(2,12)$. Las letras en minúsculas representan los valores en logaritmos naturales. Los valores entre paréntesis son el número de rezagos utilizados en cada prueba. Los valores entre corchetes indican la probabilidad de la prueba. Periodo 1965-2004.

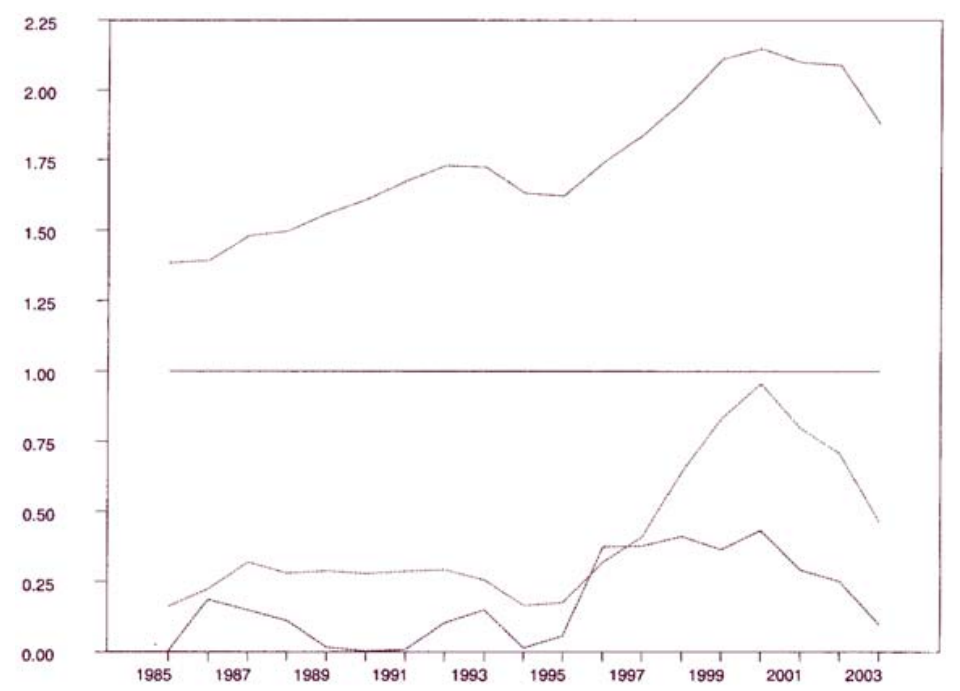

Gráficas 1A. Pruebas de estabilidad estructural del vector de cointegración. E1: $e_{t}=\beta_{1}^{*} y_{t}+\beta_{2} * p_{e}$. Estabilidad del vector de cointegración: Modelo-R. Nivel de significancia $=99 \%$. Cada línea representa el rango de cointegración, iniciando de rango cero $(r=0)$ de arriba hacia abajo hasta $\mathrm{r} \leq 2$. 


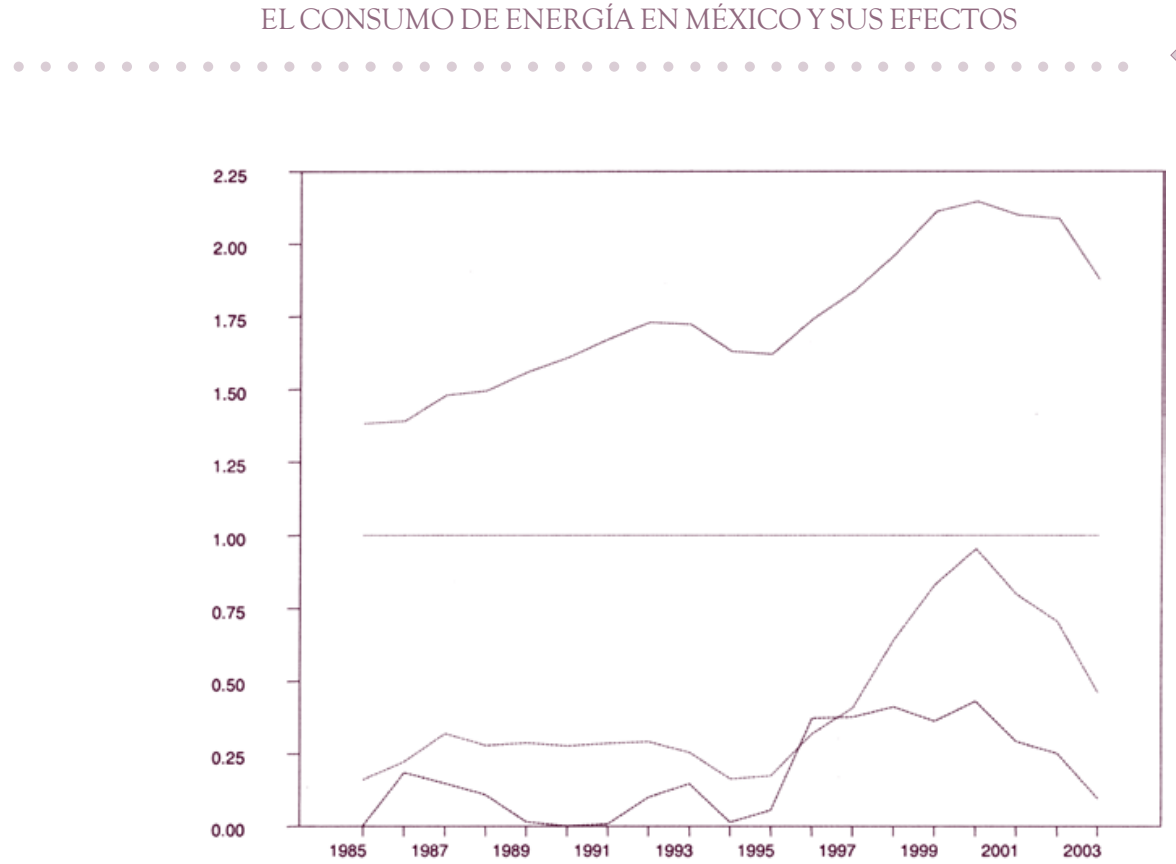

Gráficas 1Ab. Pruebas de estabilidad estructural del vector de cointegración. E1: $e_{t}=\beta_{1} * y_{t}+\beta_{2}{ }^{*} p_{e}$. Estabilidad del vector de cointegración: Modelo-Z. Nivel de significancia $=99 \%$. Cada línea representa el rango de cointegración, iniciando de rango cero $(r=0)$ de arriba hacia abajo hasta $r \leq 2$.

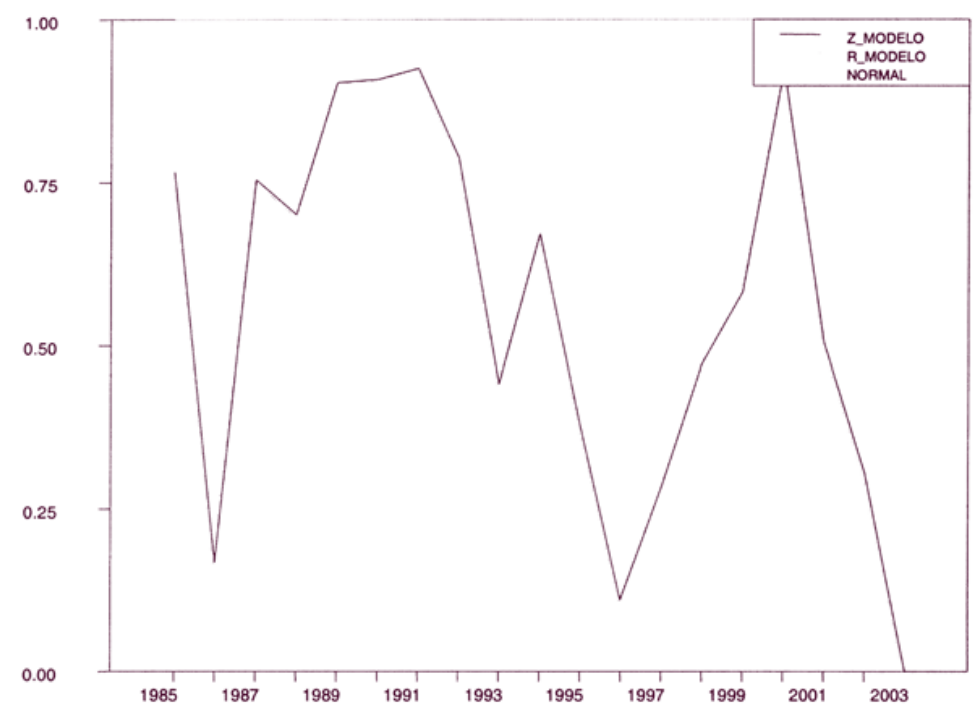

Gráficas 1Ac. Pruebas de estabilidad estructural del vector de cointegración. E1: $e_{t}=\beta_{1} * y_{t}+\beta_{2} *$ pre $_{t}$. Estabilidad de Sp (b). Nivel de significancia $=95 \%$. 


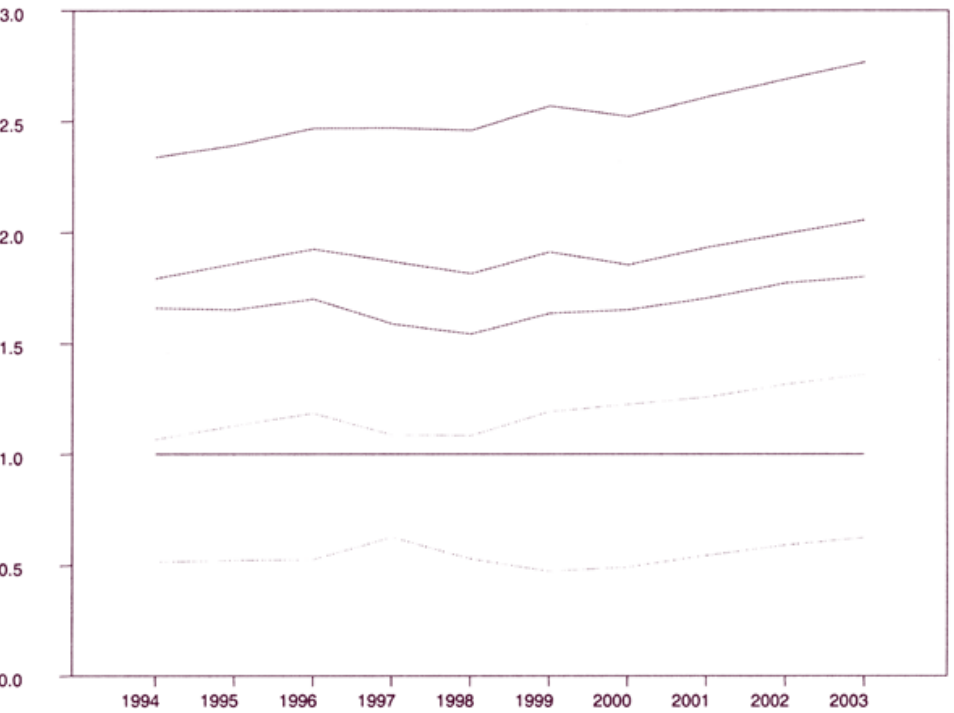

Gráficas 2A. Pruebas de estabilidad estructural del vector de cointegración. E2: $y_{t}=\beta_{1} * i_{t}+\beta *{ }_{2} t_{t}$ $+\beta_{3} * x_{t}-\beta_{4} *$ pre $_{t}$. Estabilidad del vector de cointegración: Modelo-R. Nivel de Significancia $=90 \%$. Cada línea representa el rango de cointegración, iniciando de rango cero $(r=0)$ de arriba hacia abajo hasta $\mathrm{r} \leq 4$.

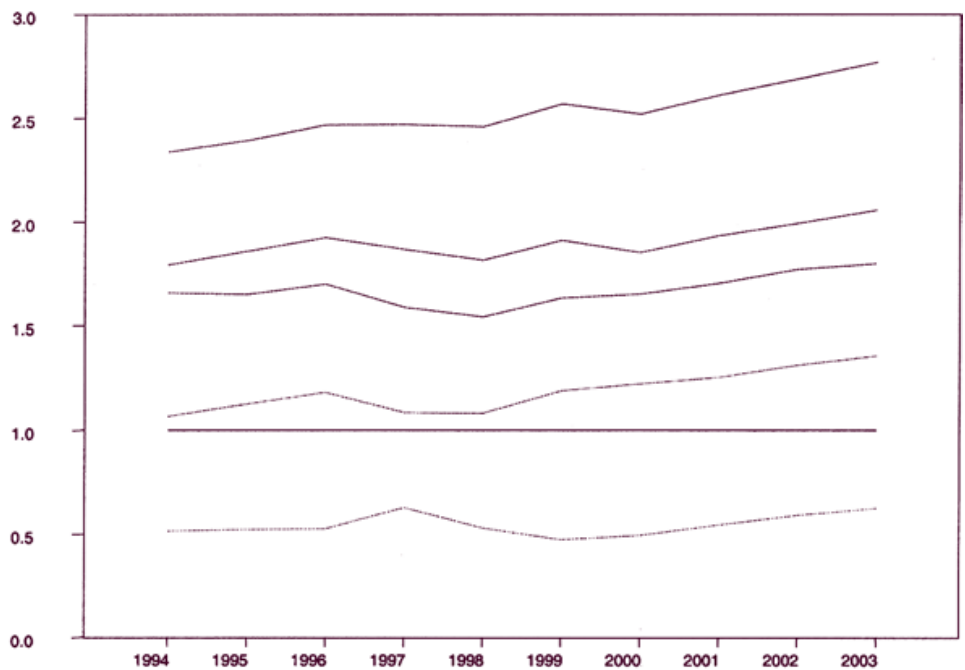

Gráficas 2Ab. Pruebas de estabilidad estructural del vector de cointegración. E2: $y_{t}=\beta_{1} * i_{t}+\beta_{2}{ }^{*} t c_{t}$ $+\beta_{3} * x_{t}-\beta_{4} *$ pre $_{t}$. Estabilidad del vector de cointegración: Modelo-Z. Nivel de significancia $=90 \%$. Cada línea representa el rango de cointegración, iniciando de rango cero $(r=0)$ de arriba hacia abajo hasta $\mathrm{r} \leq 4$. 


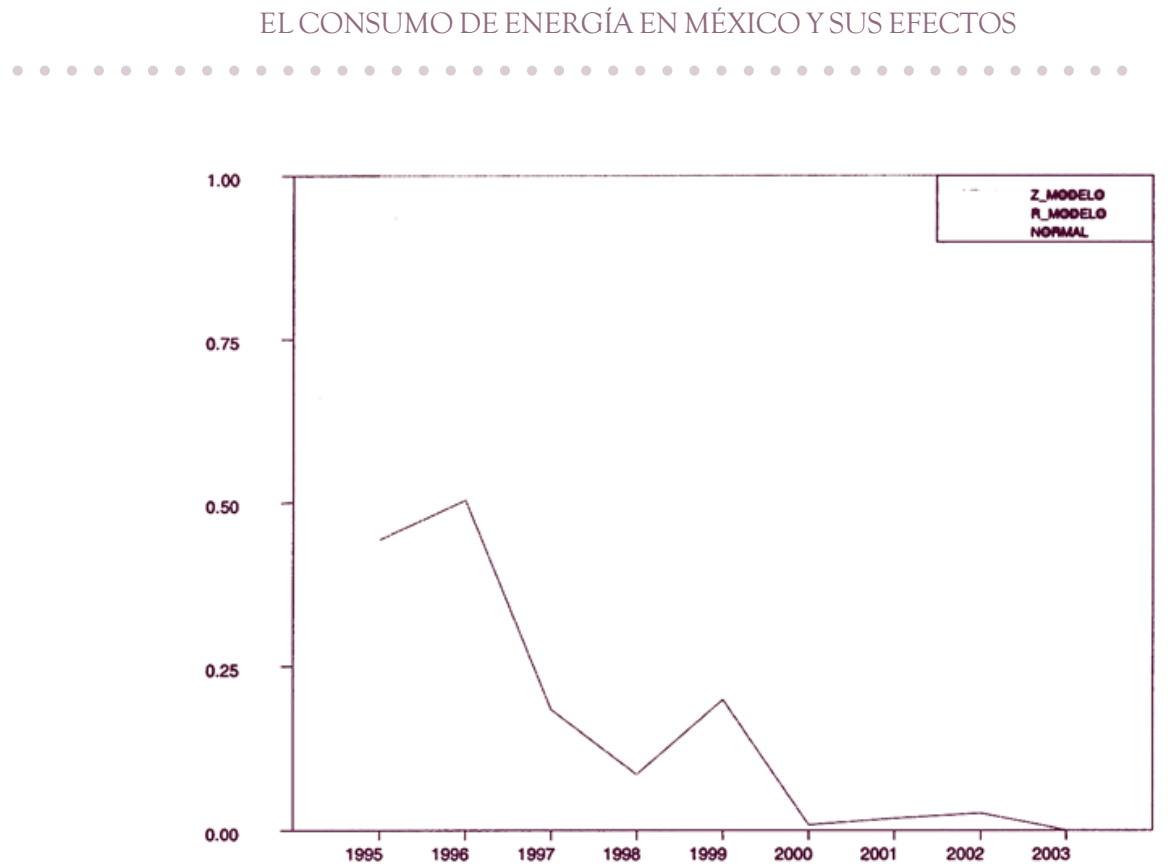

Gráficas 2Ac. Pruebas de estabilidad estructural del vector de cointegración. E2: $y_{t}=\beta_{1} * i_{t}+\beta_{2} * t_{t} r_{t}$ $+\beta_{3} * x_{t}-\beta_{4} * p_{t}$. Estabilidad de Sp(b). Nivel de significancia $=95 \%$.

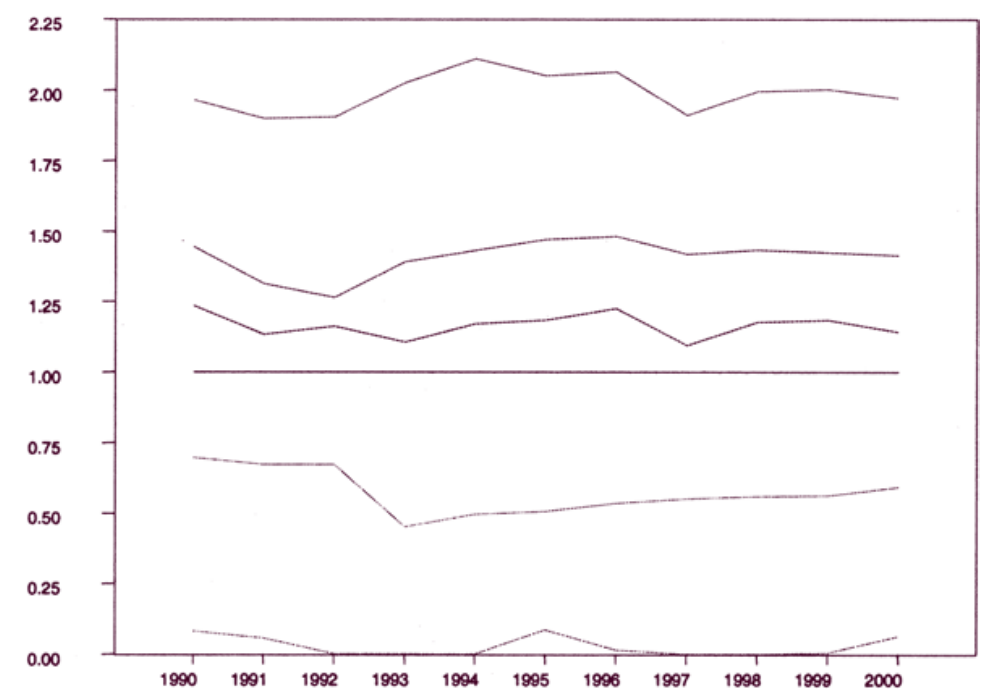

Gráfica 3A. Pruebas de estabilidad estructural del vector de cointegración E3: $\mathrm{p}_{\mathrm{t}}=\mathrm{b}_{1} * \mathrm{tc}_{\mathrm{t}}+\mathrm{b}_{2}{ }^{*} \mathrm{w}_{\mathrm{t}}$ $+b_{3}{ }^{*} 2_{t}+b_{4}{ }^{*}$ pre . Estabilidad del vector de cointegración: Modelo-R. Nivel de significancia $=90 \%$. 


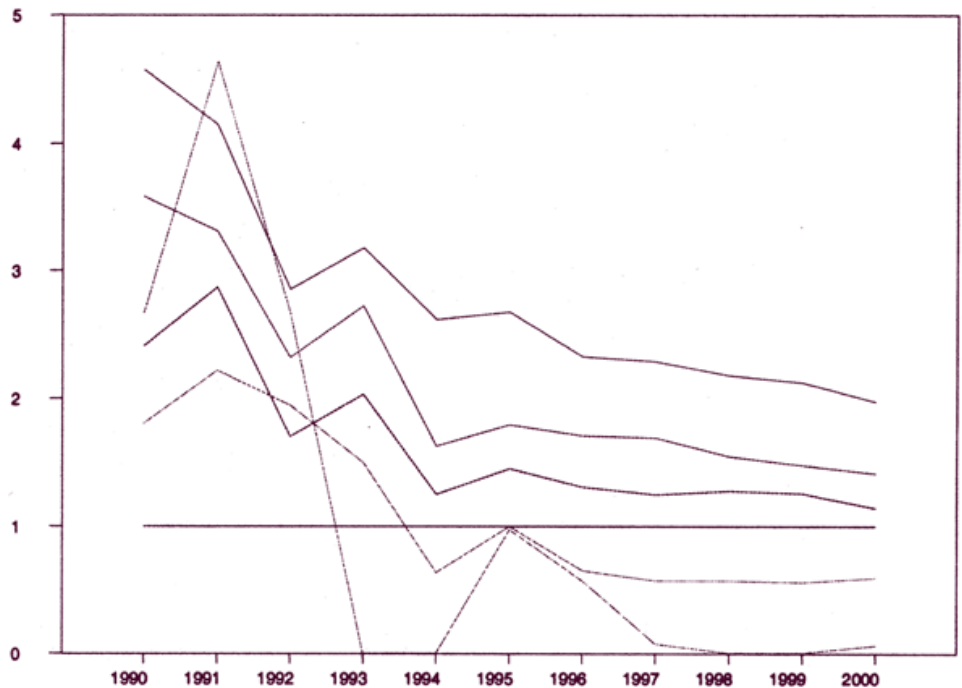

Gráficas 3Ab. Pruebas de estabilidad estructural del vector de cointegración E3: $\mathrm{p}_{\mathrm{t}}=\mathrm{b}_{1} * \mathrm{tc}_{\mathrm{t}}+\mathrm{b}_{2} * \mathrm{w}_{\mathrm{t}}$ $+\mathrm{b}_{3} * \mathrm{~m} 2_{\mathrm{t}}+\mathrm{b}_{4}{ }^{*}$ pre $_{\mathrm{t}}$. Estabilidad del vector de cointegración: Modelo-Z. Nivel de significancia $=90 \%$.

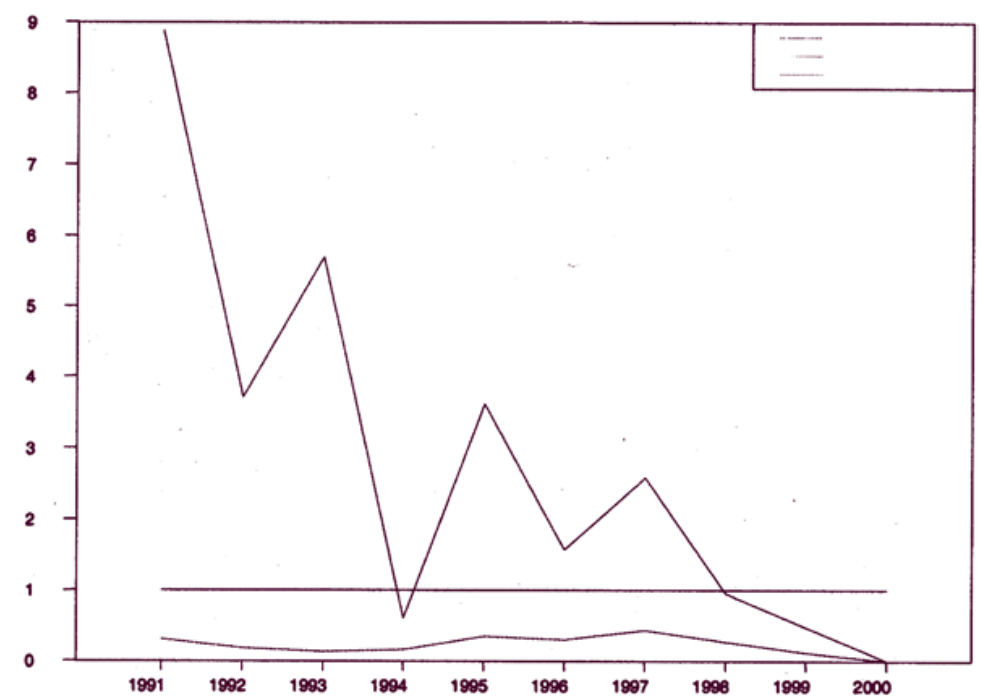

Gráficas 3Ac. Pruebas de estabilidad estructural del vector de cointegración E3: $\mathrm{p}_{\mathrm{t}}=\mathrm{b}_{1} * \mathrm{tc}_{\mathrm{t}}+\mathrm{b}_{2} * \mathrm{w}_{\mathrm{t}}$ $+b_{3}{ }^{*} m 2_{t}+b_{4} *$ pre $_{t}$. Estabilidad de Sp(b). Nivel de significancia $=95 \%$. 


\section{Descripción y fuentes de la base de datos}

$\mathrm{e}_{\mathrm{t}}=$ demanda nacional de energía (petajoules), Balance Nacional de Energía Secretaría de Energía (SENER), http://www.energia.gob.mx

$\mathrm{y}_{\mathrm{t}}=$ producto interno bruto real en millones de pesos $(1993=100)$, Instituto Nacional de Estadística, Geografia e Informática (INEGI), http://www.inegi.gob.mx

$\mathrm{p}_{\mathrm{t}}=$ índice nacional de precios al consumidor (base 2002 = 100), Índice de precios al consumidor por objeto del gasto nacional, Índice General, Banco de México (BanXICo), http:// www.banxico.org. $m x$

$\mathrm{pe}_{\mathrm{t}}=$ índice nacional de precios de la energía (base 2002=100), Índice de precios al consumidor por objeto del gasto nacional, Electricidad y Combustibles, Banco de México (BANXICO), http:// www.banxico.org. $m x$

pre $_{t}=$ precio relativo de la energía $\left(\mathrm{pe}_{\mathrm{t}} / \mathrm{p}_{\mathrm{t}}\right)$.

$\mathrm{i}_{\mathrm{t}}=$ formación bruta de capital fijo en miles de pesos (1993=100). Sistema de Cuentas Nacionales Instituto Nacional de Estadística, Geografía e Informática (INEGI), http://www.inegi.gob.mx

$\mathrm{x}_{\mathrm{t}}=$ producto de Estados Unidos en miles de millones de dólares (base 2002=100) Federal Reserve Bank of St. Louis, http://research.stlouisfed.org/fred2/

$\mathrm{tcr}_{\mathrm{t}}=$ tipo de cambio real, Banco de México (BANXICO), http://www.banxico.org.mx.

$\mathrm{s}_{\mathrm{t}}=$ tipo de Cambio Nominal, Banco de México (BANXICO), http://www.banxico.org.mx.

$\mathrm{w}_{\mathrm{t}}=$ salario mínimo general promedio, Comisión Nacional de Salarios Mínimos, http:// www.conasami.gob.mx

$\mathrm{m} 2 \mathrm{t}=$ agregado monetario M2 en miles de pesos, Banco de México (BanXico), http:// www.banxico.org. $m x$ 


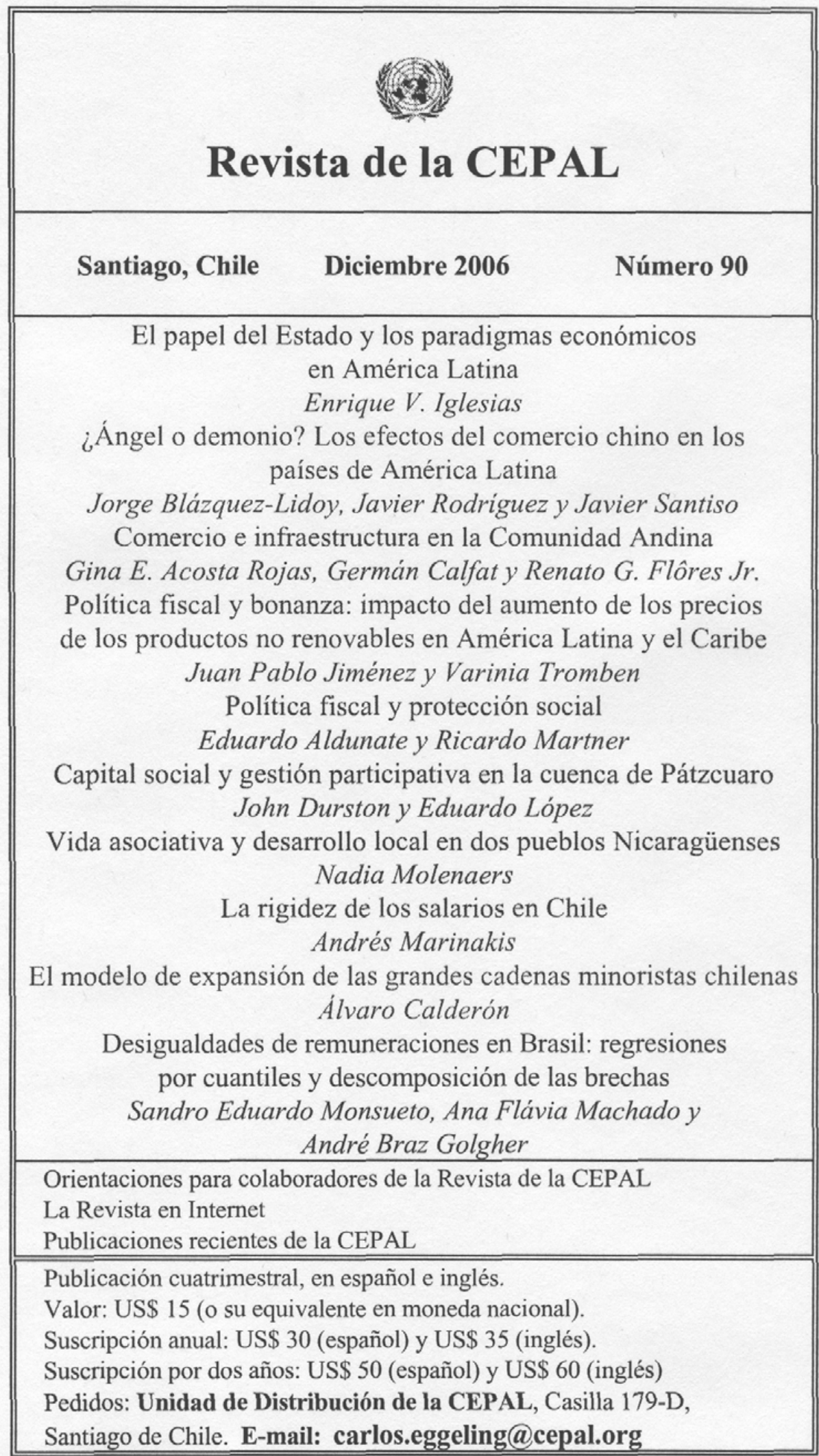

